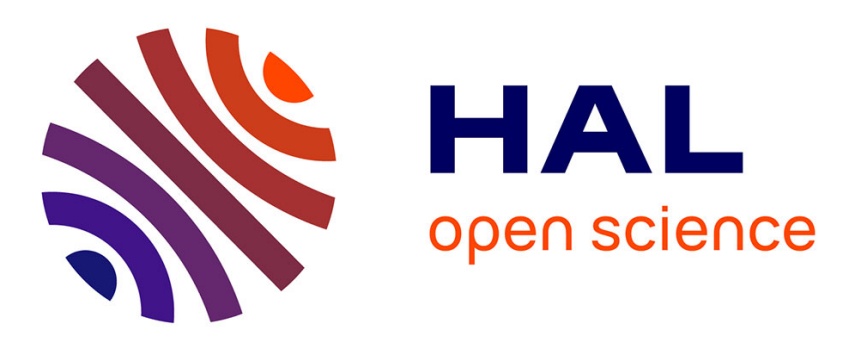

\title{
A mechanistic model to predict distribution of carbon among multiple sinks.
}

\author{
André Lacointe, Peter E.H. Minchin
}

\section{To cite this version:}

André Lacointe, Peter E.H. Minchin. A mechanistic model to predict distribution of carbon among multiple sinks.. Phloem: Methods and Protocols, Editions Springer, 473 p., 2019, Methods in Molecular Biology, 978-1-4939-9561-5. hal-02788117

\section{HAL Id: hal-02788117 https://hal.inrae.fr/hal-02788117}

Submitted on 5 Jun 2020

HAL is a multi-disciplinary open access archive for the deposit and dissemination of scientific research documents, whether they are published or not. The documents may come from teaching and research institutions in France or abroad, or from public or private research centers.
L'archive ouverte pluridisciplinaire HAL, est destinée au dépôt et à la diffusion de documents scientifiques de niveau recherche, publiés ou non, émanant des établissements d'enseignement et de recherche français ou étrangers, des laboratoires publics ou privés. 
1 A mechanistic model to predict distribution of carbon among multiple 2 sinks.

André Lacointe ${ }^{(1)}$, Peter E.H. Minchin ${ }^{(2)}$

(1) Université Clermont Auvergne, INRA, PIAF, F-63000, Clermont-Ferrand, France; email: andre.lacointe@inra.fr

(2) New Zealand Institute for Plant and Food Research, Motueka Research Centre, Motueka, New Zealand; email: Peter.Minchin@ plantandfood.co.nz

\section{Abstract/summary.}

Modelling is a fundamental part of quantitative science. It is a methodology of the holistic approach of bringing together quantitative ideas, many of which will have been developed though a reductionist approach that allows a lot of detail to be gathered on a small part of the system of interest. Phloem and xylem physiology are both descriptions of whole plant behaviour. The phloem is especially difficult to study in a reductionist way because as soon as the phloem is disturbed, even very carefully, it stops functioning by induction of blockage and other defensive mechanisms. This was the cause of a long debate on the basic structure of the phloem's long-distance transport pathway. Were the sieve-tubes 'blocked' at the sieve-plates or was there a continuous open conduit between source and sink? Developments in very rapid chilling of small pieces of phloem tissue, to obtain the required speed of cooling, was needed before reliable micrographs could be obtained and conclusively showed that the observed sieve-plate blockages were an artefact brought about by phloem damage quickly leading to blockage mechanisms, believed to be needed to prevent loss of significant phloem sap when

27 plants are damaged

It is now generally accepted that phloem flow is the result of bulk solution flow generated by osmotic pressure generated by phloem loading. But there is still little agreement on how sink competition functions and the well documented source sink relations observed with tracer studies. More recently the importance of phloem pathway leakage (unloading) and reloading 
1 has been recognised and the role of this is still being unravelled. Interactions between phloem

2 and xylem flows are now thought to be important, and may have a role in carbohydrate

3 source-sink relations through potassium recirculation.

4 All of these areas are extremely difficult to research by the reductionist approach, with

5 modelling being an important tool to test the consequences of proposed mechanisms which

6 can then be tested in whole plant experiments.

7 Phloem/xylem modelling has been at the limits of quantitative modelling, especially when

8 dynamic models are needed to explain tracer studies. Huge advances in computing now

9 enable more realistic modelling and the PiafMunch approach has extended that even further

10 by enabling much more mechanistic detail to be incorporated. With the recent introduction of

11 tracer dynamics now incorporated in PiafMunch it will be possible to look at the effects of

12 specific phloem mechanisms upon the shape of evolving tracer profiles.

15 Keywords: Münch model, carbon allocation, sink priority, phloem, xylem, coupled water and 16 carbon fluxes, plant architecture, functional - structural plant modelling, source sink relations 


\section{Introduction}

3 Wardlaw (1990) reviewed a large body of experimental data on carbon partitioning in plants

4 and found no mechanistic understanding of the data. More recently Lacointe (2000) reviewed

5 the range of models used in functional-structural tree models where he reviewed the empirical

6 methods based on allometery, sink priorities and functional equilibrium, but found no

7 mechanistic approaches to this fundamental aspect of balanced plant growth.

8

9 Currently, the general consensus of phloem flow is that proposed by Münch (1928) with bulk

10 flow of phloem sap driven by an osmotically generated pressure gradient created by loading

11 of photosynthate (usually sucrose) at the source and unloading at the sink. In many plant

12 species phloem loading is an active transport process across the sieve-tube plasmalemma

13 resulting in a source solute concentration in the order of $0.8 \mathrm{M}$ while in other species this is a

14 passive process relying on diffusional flow from the cells associated with photosynthesis.

15 Detailed biophysical models of this process were first described by Christy and Ferrier (1973)

16 and this work has had considerable complexity added resulting in the recent work of

17 Thompson and Holbrook (2003). This work describes a single-source single-sink system with

18 concomitant water flow in terms of parameters describing phloem loading, phloem unloading,

19 and includes lateral water flow through an ideal (i.e. reflection coefficient for the solute is

20 one) sieve-tube plasmalemma.

22 The first attempt to extend this approach to multiple sinks supplied by a single source was that

23 of Minchin et al. (1993). Their simple 2-sink model was able to mimic several observed

24 phenomena involving shoot-root interactions and gave the first quantitative explanation of

25 sink priority. This model predicted that the relative sink priorities between the shoot and root 
1 of a barley seedling could be reversed by cooling the root, and this was subsequently

2 demonstrated (Minchin et al., 1994). This preliminary model was based on a non-permeable,

3 either by water or solute, long-distance transport pathway which is known not to be the case.

4 This simplification greatly simplified the model equations allowing them to be solved

5 analytically. Bidel et al. (2000) expanded this approach to many sinks representing a growing

6 root and used an iterative approach to determine how carbohydrate flows were able to mimic

7 different patterns of root growth by altering the individual sink properties.

9 But it is well know that the plant vasculature consists of both phloem and xylem which are 10 physically close and readily interact through both water relations and controlled transfer of

11 solutes. Daudet et al. (2002) incorporated xylem/phloem interactions through water relations

12 which could now incorporate effects of transpiration-induced gradients of water potential.

13 Local gradients of all water- and carbon-flux related variables could be accounted for by a

14 spatially discretized approach, which turned partial differential to ordinary differential

15 equations. They used P-Spice ${ }^{\mathrm{TM}}$ software to illustrate their methods on a branching system

16 with three source leaves, and three competing fruits. This work has been extended (Lacointe

17 and Minchin, 2008) to allow huge flexibility in architecture and specific mechanistic detail

18 through use of recently developed numerical methods, resulting in the model 'PiafMunch'.

19 Recently, Hall and Minchin (2013) proposed a closed-form solution for steady-state coupled

20 phloem/xylem using the Lambert-W function, which can handle multiple sinks. While

21 incorporating some of the added complexities, such as variation of phloem resistance with

22 solute concentration, and deviations in the Van't Hoff expression for osmotic pressure, the

23 differential equation approach is still quite limited in its ability to handle a lot of detail of

24 physiological interest (e.g. pathway unloading/reloading of solute, different unloading 
1 kinetics). By contrast, a major advantage of the PiafMunch approach is its flexibility to be

2 able to work with a huge range of local loading and unloading mechanisms.

4 In this chapter, we will first describe the original PiafMunch model as published in 2008 in

5 detail. Then its capacities will be illustrated by examples of use and results. The third part will

6 introduce recent and current developments regarding $(i)$ a more general description of the

7 plant architecture, and (ii) inclusion of additional, refined biophysical or metabolic processes.

8 Finally, practical details will be given to help potential users to handle the model efficiently.

10 PiafMunch -- the original model (Lacointe and Minchin, 2008)

11 As a functional-structural model, PiafMunch includes both an architectural description of the plant structure and a mechanistic description of relevant biophysical processes at local level.

\section{Discretisation of the plant structure}

15 The plant skeleton is discretized into an arbitrary number of segments delimited by junction

$16 \operatorname{nodes}^{1}$ (Fig. 1). The plant architecture is thus represented as a collection of elements, each

17 consisting of a topological node and an associated axial pathway segment, with the exception

18 of the 'collar' node, whose physical connecting upward and downward pathway segments are

19 conventionally assigned respectively to its upper stem and lower root elements. Most

20 elements are connected to one upper and one lower element, except for the ends of roots

21 where there is no 'lower' element, the ends of stems where there is no 'upper' element, and

22 branching elements which have a single connection at one end and two at the other. Thus, a

23 total of seven different element types (denoted by different colours in Fig. 1) are required and

24 sufficient to describe any branched architecture.

\footnotetext{
${ }^{1}$ the term 'node' here is being used in the topological sense, without reference to botanical nodes which bear the leaves on a plant shoot. In future we will simply use the term node.
} 
3 Each axial pathway segment includes one phloem and one xylem pathway, which are

4 connected to each other by a transverse pathway (Fig.2) allowing for lateral water exchanges

5 between the sieve tube and local apoplasm. At end nodes, water exchanges with external

6 environment are represented, either as imposed local fluxes (e.g. measured transpiration

7 rates), or constrained by outside (e.g. soil) local water potential. Those represent the system

8 boundary conditions, which are allowed to fluctuate.

10 Hydraulic fluxes

11 According to the accepted Münch theory (1928), viscous flow of phloem sap is driven by an

12 axial hydraulic pressure gradient generated by active loading of solutes at the source and

13 unloading in the sink. Lateral solute leakage with reloading occurs along the long-distance

14 pathway, as does lateral water flow determined by water potential gradients and sieve-tube

15 membrane water permeability.

16 Xylem flow is driven by the axial apoplastic pressure gradient generated by leaf 17 transpiration.

18 These basic principles are expressed for each element as a set of equations involving 19 its own local variables and parameters. The volume fluxes of xylem and phloem water are 20 respectively

$$
J W_{X y l}=\Delta P_{X y l} / r_{X y l} \text { xylem flow between connected elements }
$$

$$
J W_{S T}=\Delta P_{S T} / r_{S T} \quad \text { phloem flow between connected elements }
$$

23 The phloem sieve tube resistance $r_{S T}$ can be either entered as a local parameter or estimated

24 from sieve tube geometry and sap viscosity (Thompson and Holbrook 2003 - see chapters 22

25 and 26 in this book). Sap viscosity is dependent on temperature and solute concentration, 
1 which is empirically described by an exponential to within $1 \%$ of experimental values over a

2 wide range of temperatures and concentrations (Gilli 1997, after Mathlouthi and Génotelle 3 1995). water potential:

6

$$
J W_{l a t}=\left(\Psi_{X y l}-\Psi_{S T}\right) / r_{l a t}
$$

7 where $r_{\text {lat }}$ is the sum of the apoplastic pathway resistance between xylem and phloem and the 8 sieve-tube cross-membrane resistance, which is inversely proportional to the membrane 9 permeability.

10 Taking into account the non-zero partial molal volume of sucrose $\bar{V}$ adds an extra 11 lateral component NZS to the volume flow into the sieve tubes:

$$
J W_{\text {lat }}=N Z S+\left(\Psi_{X y l}-\Psi_{S T}\right) / r_{l a t}
$$

$$
N Z S=\bar{V} \cdot J S_{\text {lat }}
$$

14 where $J S_{\text {lat }}$ is the lateral solute flow (see next section).

Hydrostatic pressure $P_{S T}$ within the sieve tubes is given by the difference between total phloem water potential and osmotic potential inside sieve tubes:

$$
P_{S T}=\Psi_{S T}-\Pi_{S T}
$$

18 Xylem sap has a very low solute concentration which we shall ignore, so there is no osmotic

19 component to its total water potential:

$$
P_{X y l}=\Psi_{X y l}
$$

21 For a single phloem solute, $\Pi_{S T}$ is determined by its concentration $C_{S T}$. For a dilute solution, $\Pi_{S T}$ is given by the Van't Hoff relation:

$$
\Pi_{S T}=-R T C_{S T}
$$


1 where $R$ is the universal gas constant and $T$ the absolute temperature. For a non-dilute

2 solution we use the empirical equation stated by Thompson and Holbrook (2003):

$$
\Pi_{S T}=-\rho_{w} R T\left(0.998 m+0.089 m^{2}\right)
$$

4 with $\rho_{w}$ the density of water and $m$ the molality given by

$$
m=C_{S T} /\left[\rho_{w}\left(1-C_{S T} \cdot \bar{V}\right)\right]
$$

6 If the partial molal volume of sucrose $\bar{V}$ is taken as zero then equations 5' and 5" reduces to 7 the Van't Hoff relation (5), and equation (3') reduces to the Ohm's law analog (3). When the

8 solute is sucrose and the concentration is $1 \mathrm{~mol} \mathrm{~L}^{-1}$ (typical at the site of phloem loading) then

9 using the Van't Hoff relation for a dilute solution results in about an $8 \%$ error in $\Pi_{S T}$, while at

$10 \quad 0.5 \mathrm{~mol} \mathrm{~L}^{-1}$ sucrose this reduces to a $2 \%$ error. As this is low enough in most situations

11 (compared to other error sources e.g. in the model parameter values), this refinement can be 12 deactivated by the user to reduce computation time.

14 The set of water-flow equations for each element is completed by a flow conservation

15 statement for each of the two hydraulic pathways within an element, one for the xylem and 16 one for the phloem (Fig. 2):

$$
\sum_{k} J W_{-k} \stackrel{17}{=} 0
$$

19 where $J W_{-k}$ (with appropriate sign) represents the lateral and all longitudinal liquid flows

20 to/from the node $\mathrm{k}$, the number of which depends on the element type as defined above. In

21 particular, xylem flow for terminal elements includes transpiration at stem ends ('leaves') and water uptake from soil at the root tips (Fig. 2). Note that eq. (6) assumes unchanging sieve

23 tube volume (rigid sieve-tube cell walls), which Thompson and Holbrook (2003) showed to 24 be an acceptable approximation in many situations. 
1 Solute flows

2 Longitudinal phloem solute flow between two connected elements is given by:

$$
J S_{S T}=J W_{S T} C_{S T}
$$

4 where $C_{S T}$ is the sieve-tube solute concentration in the upflow element.

6 Variation of sieve-tube solute content $Q_{S T}\left(=C_{S T} \cdot V_{S T}\right)$ is:

7

8

9

$$
\frac{d Q_{S T}}{d t}=J S_{\text {lat }}+\sum_{k} J \delta_{-} k
$$

where $J S_{-k}$ (with appropriate sign) represents all longitudinal solute flows from/to the connected element(s), and $J S_{\text {lat }}$ is the lateral solute flow into the sieve-tube.

The lateral solute flow rate $J S_{l a t}$, i.e. the local unloading/reloading, can be either set directly as an independent equation or derived from local metabolism (e.g. respiration, photosynthesis or starch $\leftrightarrow$ soluble sugar conversion) occurring in an attached parenchyma compartment. This is up to users who can write their own set of equations for $J S_{\text {lat }}$ which can have any form, including ordinary differential equations. However, a predefined set of classical equations is proposed for convenience :

$$
J S_{\text {lat }}=k_{1} \cdot\left(C_{P a r}-C_{S T}\right) \cdot V_{S T}+\left(k_{2} C_{P a r}+k_{3}\right) V_{P a r}
$$

where $C_{P a r}$ is the parenchyma solute concentration, $V_{S T}$ the sieve-tube volume and $V_{P a r}$ the parenchyma volume. This allows a number of different dynamics by assigning specific values to local parameters $k_{1}, k_{2}, k_{3}, e . g$. :

- diffusion-like kinetics $\left(k_{2}=k_{3}=0\right)$; - constant loading/unloading $\left(k_{1}=k_{2}=0\right)$; - concentration-dependent loading with a target concentration $C_{\text {targ }}$

$$
\left(k_{2}=-k_{1} V / V_{\text {Par }}, k_{3}=k_{1} C_{\text {targ }} V_{S T} / V_{\text {Par }}\right) ;
$$


24 allowing simulation of a number of dynamics, e.g. Michaelis-Menten kinetics for synthesis

- concentration-dependent unloading as in Thompson and Holbrook (2003)

$$
\left(k_{3}=0, k_{2}=-k_{1} V_{S T} / V_{P a r}\right)
$$

such that simple cases of symplastic loading/unloading are currently built into equation (9).

The default equation for parenchyma solute content $Q_{P a r}\left(=C_{P a r} \cdot V_{P a r}\right)$ change rate simulates the result of sucrose exchange with local sieve tubes $\left(J S_{\text {lat }}\right.$, eq. 9$)$, exchange with environment (maintenance respiration and/or photosynthesis) and starch/sucrose interconversion:

$$
\frac{d Q_{\text {Par }}}{d t}=-J S_{\text {lat }}-R_{M}+P h-\frac{8 l S}{d t}
$$

where respiration $R_{M}$, photosynthesis $P h$, and starch $S$ are all expressed in sucrose equivalents. The dynamics of photosynthesis $P h$ may be either read from an external file or modelled by the user $e . g$. as a periodic function (Daudet et al., 2002). For maintenance respiration $R_{M}$, the proposed formalism is that of Thornley (1970; see review by Le Roux et al., 2001), with a concentration-dependent maintenance coefficient to account for phloem sucrose leakage / active reloading:

$$
R_{M}=\left(k_{4}+k_{5} C_{S T}\right) S r
$$

where $\mathrm{Sr}$ is the structural carbon content of the element biomass, expressed in sucrose equivalents.

The default representation of starch metabolism uses a general equation derived from Daudet et al. (2002):

$$
\frac{d S}{d t}=\frac{v_{\max } \cdot C_{P a r}}{k_{M}+C_{P a r}} \cdot V_{P a r}-k_{h y d} \cdot S+k_{6} \cdot\left(C_{P a r}-C_{t a r g}\right) \cdot V_{P a r}^{2}
$$

$$
\text { from a sucrose substrate (through parameters } v_{\max } \text { and } k_{M} \text { ), starch content-dependent }
$$


1 hydrolysis back to sucrose (parameter $k_{h y d}$ ) or sucrose concentration-dependent

2 interconversion with a target concentration $C_{\text {targ }}$.

4 All solute-fluxes equations are fully editable, with a possible redefinition of all predefined 5 parameters or definition of new ones, allowing e.g. very simple configurations like Minchin et 6 al. (1993) or Thompson and Holbrook (2003) which were used to test the model (Table 2). If

7 edited, it is up to the user to make sure that the equations make sense. By contrast, all water-

8 fluxes equations, which are the heart of the model, are hard coded except for parameters. The

9 full system is coded in $\mathrm{C}++$ and solved by a combination of LAPACK linear algebra package

10 (Anderson et al., 1999) with the sparse extension TAUCS (Toledo, 2003 ) and SUNDIALS

11 algebraic/differential equation solver package (Hindmarsh et al., 2005). The software includes

12 a graphic user interface to specify the architecture, parameters, initial values and other

13 settings. This allows use of the software, with the default equations, without having to

14 recompile it. More flexibility can be achieved be editing the solute fluxes equations and 15 recompiling.

17 Examples of what the PiafMunch model has been used on

18 The first application of the PiafMunch software was to a single-source single-sink linked by a

$195 \mathrm{~m}$ long distance pathway consisting of a tube of $7.5 \mu \mathrm{m}$ diameter conduit with membrane

20 permeable to water but not to the solute (sucrose), i.e. a 'perfect' semipermeable membrane,

21 with uniform loading along the first $0.5 \mathrm{~m}$ and unloading along the last $0.5 \mathrm{~m}$. This example was chosen for direct comparison with the work of Thompson and Holbrook (2003) using a

23 continuous differential equation framework. With the PiafMunch approach we started with

$24 \mathrm{~N}=3160$ elements and then looked at the effect of reducing $\mathrm{N}$ to a much smaller number.

25 With the large value of $\mathrm{N}$ the phloem water flux was very similar to that calculated by the 
1 continuum approach of Thompson and Holbrook (2003), with the greatest differences being

2 about 2\% when the fluxes were changing at the greatest rate (see Lacointe and Minchin 2008,

3 Fig.3). That low $2 \%$ discrepancy can be ascribed to the variation of lumen diameter with

4 pressure, which was included in Thompson and Holbrook (2003) but ignored in PiafMunch.

5 With much lower values of the element number $\mathrm{N}($ e.g. $\mathrm{N}=30)$, the two approaches differed

6 most at the sites of most rapid flux change with differences ca. $10 \%$, and were very close in

7 the regions along the long-distance pathway where the water flux was not rapidly changing.

8 When these comparisons were made $24 \mathrm{hr}$ into the simulation when the flows had reached

9 equilibrium, there was only a small difference between the two approaches, even with $\mathrm{N}$ as

10 low as 10 , with a maximum deviation below $10 \%$. From this it was concluded that the

11 descrete approach of the PiafMunch model resulted in similar results to the continuous

12 method of Thompson and Holbrook (2003), and that the number of discrete elements required

13 for a good approximation of the continuous system does not need to be very high.

15 The variation of sap viscosity with sugar concentration was first introduced by Bancal and

16 Soltani (2002) in a simplified Münch model, assuming uniform concentration along the

17 pathway and ignoring membrane permeability. A few years later, Hölttä et al. (2006)

18 introduced the viscosity change in a more realistic model but ignoring the specific partial

19 molal volume of sugar. In both studies, the authors concluded that high sugar loading rates

20 could block phloem transport due to high sap viscosity. PiafMunch incorporates that variation

21 in sap viscosity with solute concentration as well as the partial molal volume of the solute;

22 these refinements can be set on/off by a simple click, either individually or both together. It

23 was confirmed that both of these had significant effect, both on the equilibrium and non-

24 equilibrium flux. Work is needed to determine if these effects have any physiological

25 significance. Hölttä et al. (2006) also showed that transpiration rate can be expected to 
1 interact with phloem flow, via water relations described by equation 3 above. This was also

2 demonstrated in the initial PiafMunch work (Lacointe and Minchin 2008, Fig. 8).

4 The PiafMunch model is meant to handle more complex source-sink configurations. Working

5 with a considerably simplified model with a solute molal volume of zero, constant sap

6 viscosity with changes in solute content, and a non-permeable long-distance transport conduit,

7 Minchin et al. (1993) developed a Munch model describing flow between 1-source and 2-

8 sinks which predicted changes in the proportion of total solute flow delivered to each sink

9 when the source supply changed, which in experimental work has been described in terms of

10 sink priority. This was the first mechanistic description of sink priority. Working with the

11 same source sink configuration, and incorporating non-zero pathway permeability the

12 PaifMunch model showed the similar priority behaviour. The main purpose of this work was

13 to determine if the PiafMunch model gave results consistent with previous work, and it passed

14 this test with flying colours so we can have confidence in this new approach and now

15 investigate examples the previous methods cannot handle.

17 Thorpe et al. (2011) went on to model a 2-source 3-sink configuration generated in a heavily

18 pruned dwarf bean plant and test the predictions using ${ }^{11} \mathrm{C}$ tracer. Several observed treatment

19 responses were successfully predicted, but the observations could not be completely explained

20 when the modelled common pathway, comprising the stem, contained just one phloem

21 pathway. Bidirectional flow within the stem was necessary to explain the observed flows.

22 It is now accepted that the long-distance phloem transport pathway is leaky, and also takes up

23 solute from the immediate apoplast. This manifests itself in tracer studies through the

24 observed buffering of phloem flow when the phloem pathway is disturbed or there are sudden

25 changes in source or sink function. The PiafMunch model has been used to determine if this 
1 leakage/reloading alters source sink dynamics (Minchin and Lacointe, 2017). This modelling

2 indicated that the phloem flow does not follow Poiseuille dynamics, i.e. the water flux was

3 not proportional to $\Delta \mathrm{P}$, due to there always being water flow across the membrane, even

4 without pathway unloading and or reloading of solute. At equilibrium, the presence of

5 unloading altered the solute concentration and hydrostatic pressure profiles. With adequate

6 reloading along the pathway the effects of pathway unloading were completely compensated

7 for, making the equilibrium system look like one with no pathway unloading. Further work is

8 needed here to look at the non-equilibrium flows. To do this even more model parameters are

9 needed, though this might be a means of estimating these parameter values through

10 optimising the parameters to produce behaviour similar to that seen in plant experiments.

\section{Current developments}

13 A new version has been developed (PiafMunch v.2) which features a few major 14 improvements:

(1) The extension of the architectural pattern from branched to any network architecture, including loops and nodes of any connectivity order (that was limited to 3 in v.1, i.e. each node could be connected to 3 other nodes at most). That significantly extends the scope of the model, to $e . g$. the looped nervation pattern of an isolated leaf, or non-binary, verticillate branching patterns as exhibited by conifers.

(2) A significant refinement of the local tissue model at the node level (Fig. 3 and 4), with explicit apoplasmic, possibly solute-containing, compartments attached to both phloem (in addition to the sieve tube) and lateral parenchyma (in addition to symplasm). In particular, the lateral pathway from xylem vessel to phloem sieve tube $\left(r_{\text {lat }}, J W_{\text {lat }}\right.$ in eq. 3$)$ is now explicitly segmented into an apoplasmic pathway $\left(r_{T r s v}\right.$, $\left.J W_{T r s v}\right)$ and the cross-membrane pathway from phloem apoplasm to sieve tube ( $r_{P h l M b}$, 


\section{Concluding remarks}

$\left.J W_{P h l M b}\right)$. Water and solute fluxes between adjacent compartments are described by equations similar to (1-9) above, allowing more realistic simulation of e.g. apoplasmic (un)loading and related cross-membrane processes, or a convective component of symplasmic (un)loading.. Again, all parameters and solute flux equations can be redefined or edited to reduce the extended model to v.1 (Lacointe and Minchin 2008), or the simpler Minchin et al. (1993).

(3) User-defined sharp parameter changes can be implemented at specific time points, in addition to continuous parameter changes which were already possible in v.1 (with concentration-dependent phloem viscosity as a built-in example). This allows simulation of e.g. cold blocking / unblocking of the phloem pathway or aquaporin function - related changes in cross-membrane resistances (Steppe et al, 2012).

(4) Lateral parenchyma symplasmic volumes are now considered variables that can be driven by differential equations involving any other variables like local pressure, allowing simulation of $e$.g. reversible, elastic volume changes, i.e. water capacitance, involved in reversible stem diameter changes (Steppe et al., 2012), or plastic, irreversible growth (Lockhart, 1965; Daudet et al., 2002).

(5) Tracer analysis facilities have been included as a helper to design and analyse results from tracer experiments like that of Thorpe et al. (2011). They involve equations similar to the solute-related equations with additional terms for radioactive decay, in particular for ${ }^{11} \mathrm{C}$ which has a very short half-life of $c a .20 \mathrm{~min}$.

PiafMunch has proved highly efficient and reliable on simple systems -- even though they could be more complex than those modelled by other approaches in literature. However, it is meant to handle truly complex architectures and detailed local processes, using highly 
1 efficient, state-of-the-art numerical methods. It can be used to simulate and test effects of any

2 known or hypothetical mechanism, both at the local and at the global, plant-wide scale level.

3 It can be used also to simulate coupled solute/water relations of a single, isolated organ, e.g.

4 an isolated leaf, given appropriate boundary conditions dynamics. Furthermore, it can be

5 easily extended, as shown by the development of v.2. This could be readily further extended

6 to e.g. a vacuolar compartment with specific aquaporins mediating tonoplast resistances;

7 another possible extension, though slightly more difficult to implement, would be to introduce

8 multiple phloem pathways as suggested by results from Thorpe et al (2011). It should be

9 emphasized, however, that any extension involves additional parameters (theoretically $\mathrm{N}$

10 additional values for each additional local parameter, although it is often reasonable to assume

11 a common, single value), which can result in unreasonable, and confusing, complexity. This

12 can be handled by setting unnecessary parameters to zero or infinite values to keep focus on

13 those relevant to the issue of interest. Because of the multiplicity of parameters, it is

14 unrealistic to use the model as a means to optimise parameters to fit experimental data;

15 however, this could be made possible by setting a significant subset of parameters to known,

16 reasonable values and optimising only another, limited, focus subset of parameters. This

17 emphasizes the importance of membrane-focused and other experimental studies at the tissue

18 or cell level to provide such required parameter values. As for all modelling, its use is in

19 testing ideas and never a substitute to experiment.

21 Potential users interested in implementing PiafMunch in their work are very welcome to contact the authors. We will be pleased to help, either for specific applications or for practical installation details. 
1 Table 1. Variables involved in the model PiafMunch v.1 (2008)

2 Symbol Meaning unit

$3 C_{S T}, C_{P a r} \quad$ sucrose molar concentration (sieve tube, parenchyma) $\mathrm{mmol} \mathrm{mL}^{-1}$

$4 S_{\text {lat }} \quad$ lateral sucrose flux $\mathrm{mmol} \mathrm{h}^{-1}$

$5 \quad J S_{S T}{ }^{(1)} \quad$ longitudinal phloem sucrose flow between adjacent elements $\quad \mathrm{mmol} \mathrm{h}^{-1}$

$6 \quad J S_{-k} \quad$ longitudinal sucrose flow between current node and node \#k $\mathrm{mmol} \mathrm{h}^{-1}$

$7 \quad J W_{\text {lat }} \quad$ transverse water flux $\quad \mathrm{mL} \mathrm{h}^{-1}$

$8 \quad J W_{-} \quad$ lateral or longitudinal solution flow

between current node and node \#k $\mathrm{mL} \mathrm{h}^{-1}$

$10 J W_{X y l}, J W_{S T}^{(1)}$ longitudinal water flux (xylem, sieve tube) $\quad \mathrm{mL} \mathrm{h}^{-1}$

$11 m$ solution molality $\quad \operatorname{mol}(\mathrm{kg} \text { of water })^{-1}$

$12 P_{S T}, P_{X y l} \quad$ hydrostatic pressure (sieve tube, xylem) $\quad \mathrm{MPa}$

$13 Q_{S T}, Q_{P a r} \quad$ quantity of sucrose (sieve tube, parenchyma) mmol

$14 R_{M} \quad$ maintenance respiration $\quad \mathrm{mmol}_{\text {sucrose }} \mathrm{h}^{-1}$

$15 \mathrm{Sr} \quad$ structural carbon content $\mathrm{mmol}_{\text {sucrose equivalent }}$

$16 S$ starch content $\mathrm{mmol}_{\text {sucrose equivalent }}$

$17 V_{S T}, V_{P a r} \quad$ volume (sieve tube, parenchyma) $\mathrm{mL}$

$18 \quad$ NZS non-zero sugar volume flow accompanying $J S_{\text {lat }} \quad \mathrm{mL} \mathrm{h}^{-1}$

$19 \Pi_{S T} \quad$ osmotic potential $\quad \mathrm{MPa}$

$20 \Psi_{X y l}, \Psi_{S T} \quad$ water potential (xylem, sieve tube) $\quad \mathrm{MPa}$

$23{ }^{(1)} J S_{S T}, J W_{S T}$ were called resp. $J S_{P h l}, J W_{P h l}$ in Lacointe and Minchin 2008 
1 Table 2. Model parameters

\begin{tabular}{|c|c|c|}
\hline$\underline{\text { Symbol }}$ & Meaning (equation. involved) & value as used to simulate Thompson \& Holbrook 2003 \\
\hline$C_{\text {targ }}$ & target sucrose concentration for starch metabolism (eq. 12) & $0.1 \mathrm{mmol} \mathrm{mL} \mathrm{m}^{-1}$ (1) \\
\hline$k_{1}$ & \multirow{3}{*}{ lateral carbon flow rate parameters (eq. 9) } & $3.23994 \mathrm{~h}^{-1}$ in unloading zone ; 0 elsewhere \\
\hline$k_{2}$ & & $-3.23994 \mathrm{~h}^{-1}$ in unloading zone ; 0 elsewhere \\
\hline$k_{3}$ & & $0 \mathrm{mmol} \mathrm{mL} \mathrm{m}^{-1} \mathrm{~h}^{-1}$ \\
\hline$k_{4}$ & \multirow[b]{2}{*}{ Maintenance respiration - related parameters (eq. 11) } & $0 \mathrm{~h}^{-1}$ \\
\hline$k_{5}$ & & $0 \mathrm{~mL} \mathrm{mmol}^{-1} \mathrm{~h}^{-1}$ \\
\hline$k_{6}$ & Starch metabolism - related parameter (eq. 12) & $0 \mathrm{~h}^{-1}$ \\
\hline$k_{\text {hyd }}$ & relative rate of starch hydrolysis (eq. 12) & $0 \mathrm{~h}^{-1}$ \\
\hline$k_{M}$ & Michaelis-Menten constant for starch synthesis (eq. 12) & $0 \mathrm{mmol}_{\text {sucrose }} \mathrm{mL}^{-1} \quad$ (1) \\
\hline$P h$ & rate of photosynthesis (eq. 10) & $0 \mathrm{mmol}_{\text {sucrose }} \mathrm{h}^{-1}$ \\
\hline$R$ & universal gas constant (eqs. 5, 5') & $0.0083143 \quad \mathrm{MPa} \mathrm{mL} \mathrm{K}^{-1} \mathrm{mmol}^{-1}$ \\
\hline$r_{\text {lat }}$ & lateral hydraulic flow resistance (eq. 3, 3') & $23.5785 \times \mathrm{N}^{(3)} \quad \mathrm{MPah} \mathrm{mL}^{-1}$ \\
\hline$r_{S T}^{(4)}$ & axial phloem sieve tube hydraulic flow resistance (eq. 2) & $14050.3 / \mathrm{N}^{(3)} \quad \mathrm{MPa} \mathrm{h} \mathrm{mL}{ }^{-1}$ for $C_{S T}=0.5 \mathrm{mmol} \mathrm{mL}^{-1}(4)$ \\
\hline$r_{X y l}$ & axial xylem hydraulic flow resistance (eq. 1) & $10^{-200} / \mathrm{N}^{(3)} \quad \mathrm{MPa} \mathrm{h} \mathrm{mL}^{-1}$ \\
\hline
\end{tabular}




\begin{tabular}{|l|l|l|}
\hline$T$ & absolute temperature (eqs. 5, 5') & $293 \mathrm{~K}$ \\
\hline $\bar{V}$ & partial molal volume of sucrose (eqs. 3', 5”) & $0.2155 \mathrm{~mL} \mathrm{mmol}^{-1}$ \\
\hline$v_{\max }$ & kinetic parameter for starch synthesis (eq. 12) & $0 \mathrm{mmol}_{\text {sucrose equ. } \mathrm{mL}^{-1} \mathrm{~h}^{-1}}$ \\
\hline$\rho_{w}$ & density of pure water (eqs. 5', 5”) & $0.99803 \mathrm{~kg} \mathrm{~L}^{-1}$ at $T=293 \mathrm{~K}$ \\
\hline
\end{tabular}

${ }^{1)}$ software default value, without effect in simulating either Thompson \& Holbrook (2003) or Minchin et al (1993).

2 (2) user-defined input variable (does not have to be constant).

$3{ }^{(3)}$ axial (resp. lateral) hydraulic resistances are proportional (resp. inverse proportional) to element length, hence the scaling by $\mathrm{N}$ (see text).

$4{ }^{(4)} r_{S T}$ (called $r_{P h l}$ in Lacointe and Minchin 2008) changes with $C_{S T}$, in proportion to the solution viscosity. 


\section{References}

3 Anderson E, Bai Z, Bischof C, Blackford S, Demmel J, et al. (1999). 'LAPACK users' guide. 3rd edn. http://www.netlib.org/lapack/lug/index.html (accessed 28 March 2018).

6 Bancal P, Soltani F (2002). Source-sink partitioning. Do we need Münch? Journal of Experimental Botany 53, 1919-1928.

Bidel LPR, Pages L, Riviere LM, Pelloux G Lorendeau JY (2000). Mass Flow Dyn 1: A carbon transport and partitioning model for root system architecture. Annals of Botany 85, 869-886.

Christy A.L, Ferrier JM (1973). A mathematical treatment of Munch's pressure-flow hypothesis of phloem translocation. Plant Physiology 52, 531-538.

Daudet FA, Lacointe A, Gaudillère JP, Cruiziat P (2002). Generalized Münch coupling between sugar and water fluxes for modelling carbon allocation as affected by water status. Journal of Theoretical Biology 214, 481-498.

Gilli R (1997). Evaluation de différentes données physico-chimiques relatives aux solutions sucrées. http://www.associationavh.com/fr/feuilles.html (accessed 28 March 2018).

Hall AJ, Minchin PEH (2013). A closed-form solution for steady-state coupled phloem/xylem flow using the Lambert-W function. Plant, Cell and Environment 36, 2150-2162. 
1 Hindmarsh AC, Brown PN, Grant KE, Lee SL, Serban R, Shumaker DE, Woodward CS

Minchin PEH, Thorpe MR, Farrar JF (1993). A simple mechanistic model of phloem transport which explains sink priority. Journal of Experimental Botany 44, 947-955. 
1 Minchin PEH, Farrar JF, Thorpe MR (1994). Partitioning of carbon in split roots of barley:

Minchin PEH, Lacointe A (2017). Consequences of phloem pathway unloading/reloading on dwarf bean: a 2-source- 3-sink system. Functional Plant Biology 38, 127-138.

24 Toledo S (2003). TAUCS, a library of sparse linear solvers.

25 http://www.tau.ac.il/ stoledo/taucs (accessed on 28 March 2018) 
$-23-$

1

2 Wardlaw IF (1990). The control of carbon partitioning in plants. New Phytologist 116, 3413381. 


\section{Figure captions}

3 Figure 1. Representation of plant architecture. In this example, a plant (A) with 2 roots and 3

4 branches each terminated by a leaf, is represented by 18 elements (B) : one collar element

5 (blue, No. 1), six non-branched stem elements (brown; No. 2, 3, 4, 5, 6, 7), three shoot

6 terminations (green; No. 8, 9, 10), two branched stem elements (orange; No. 11, 12), 1

7 branched root element (black; No. 13), three non-branched root elements (dark grey; No. 14,

8 15, 16) and two root tips (light grey; No. 17, 18). Reproduced from Lacointe et al. (2008)

9 with permission from CSIRO Publishing.

11 Figure 2. Network of hydraulic pathways for the example presented in Fig.1. The colour

12 coding is the same as in Fig. 1. Reproduced from Lacointe et al. (2008) with permission from 13 CSIRO Publishing.

15 Figure 3. The PiafMunch v.2. extended volume flow model. $r_{\ldots}, J W_{\ldots}, P_{\ldots}, \Psi_{\ldots}, \Pi_{\ldots}, V_{\ldots}$ : resp., 16 hydraulic resistance, volume flux, turgor pressure, water potential, osmotic potential, volume;

17 for resp. (subscripts): ${ }_{S T}$, Xyl, Trsv, Apo, PhlMb, ParMb, ParApo, Sympl: sieve tube, xylem, transverse

18 (xylem to phloem) apoplasm, pathway, phloem to lateral parenchyma apoplasm, pathway,

19 sieve tube plamalemma, lateral parenchyma plasmalemma, lateral parenchyma apoplasm,

20 lateral parenchyma symplasm, $N Z S, N Z S_{\text {Par: }}$ : non-zero sugar volume flow, resp. into sieve tube

21 and parenchyma symplasm. Operator $\Delta$ means '[in] - [out]' when applied to node-to-node-

22 connector variables $\left(J W_{S T}, J W_{X y l}\right)$, or '[upflow] - [downflow]' when applied to node variables

$23\left(P_{S T}, P_{X y l}\right)$.

25 Figure 4. The PiafMunch v.2. extended solute flow model. $J S_{\ldots,}, Q_{\ldots}, C_{\ldots}, V_{\ldots}$ : resp., solute 
1 flux, total solute content, solute concentration, volume; for resp. (subscripts): $S T, A p o, P h l M b$,

2 ParMb, ParApo, Sympl: sieve tube, phloem to lateral parenchyma apoplasm, pathway, sieve tube

3 plamalemma, lateral parenchyma plasmalemma, lateral parenchyma apoplasm, lateral

4 parenchyma symplasm. Operator $\Delta$ means '[in] - [out]'. 
- 26 -

$1 \quad$ Figure 1

2

3
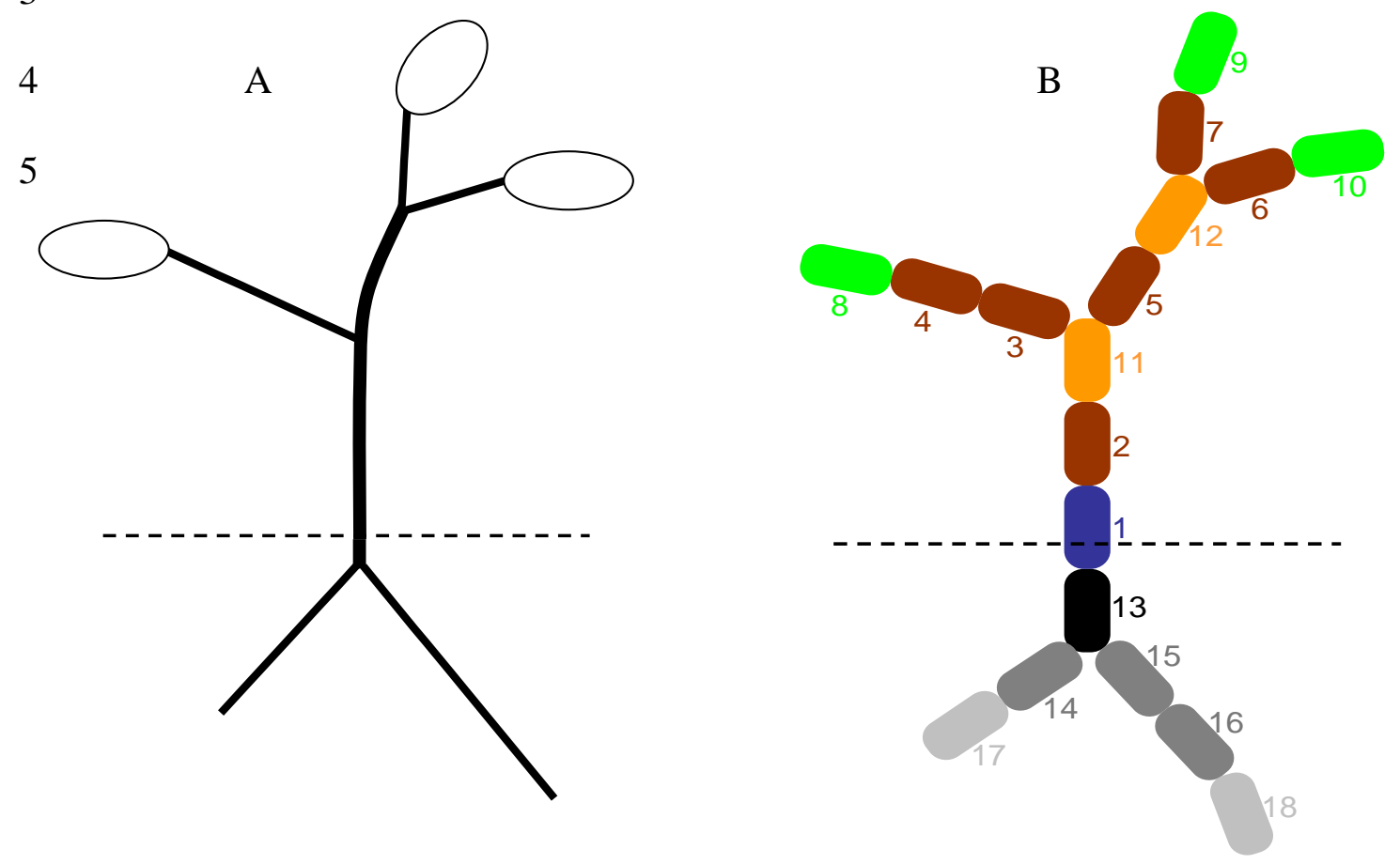
1 Figure 2.

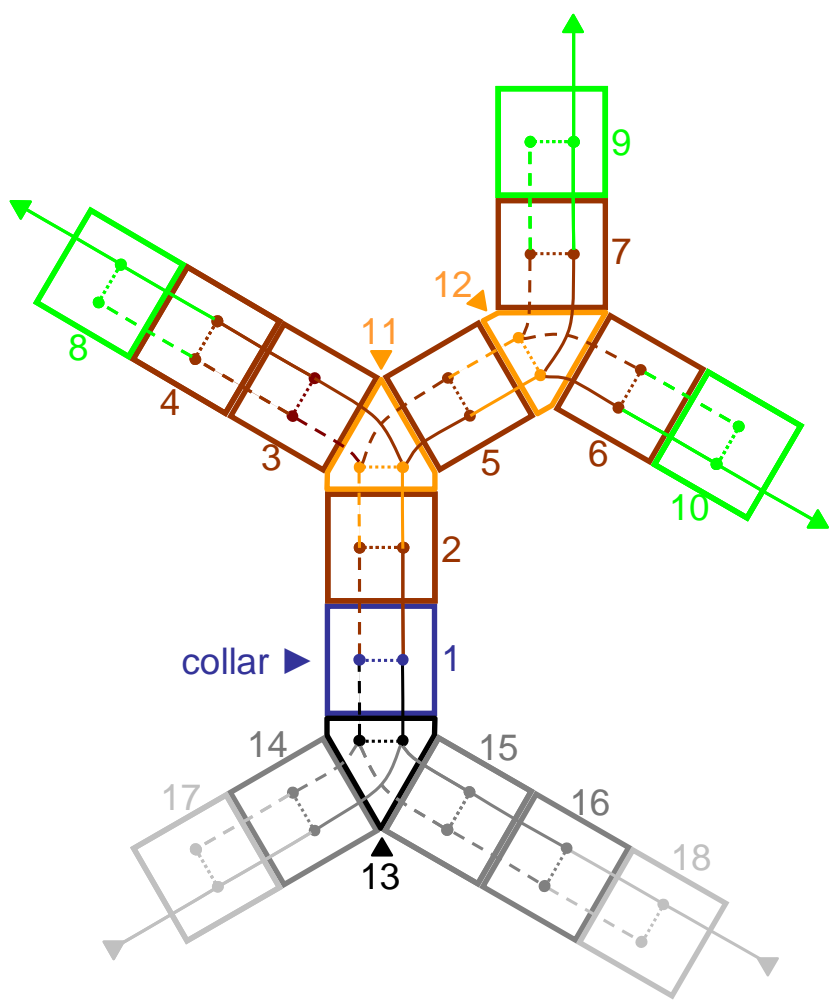

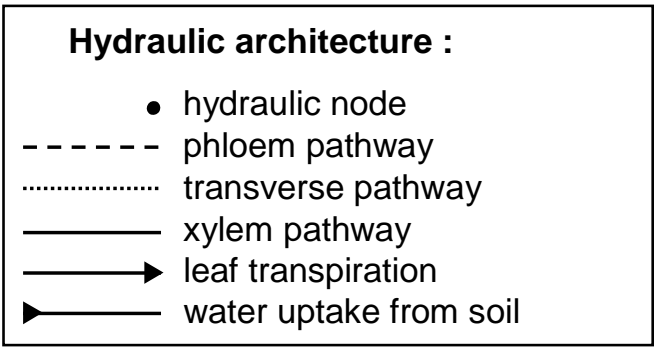


$1 \quad$ Figure 3

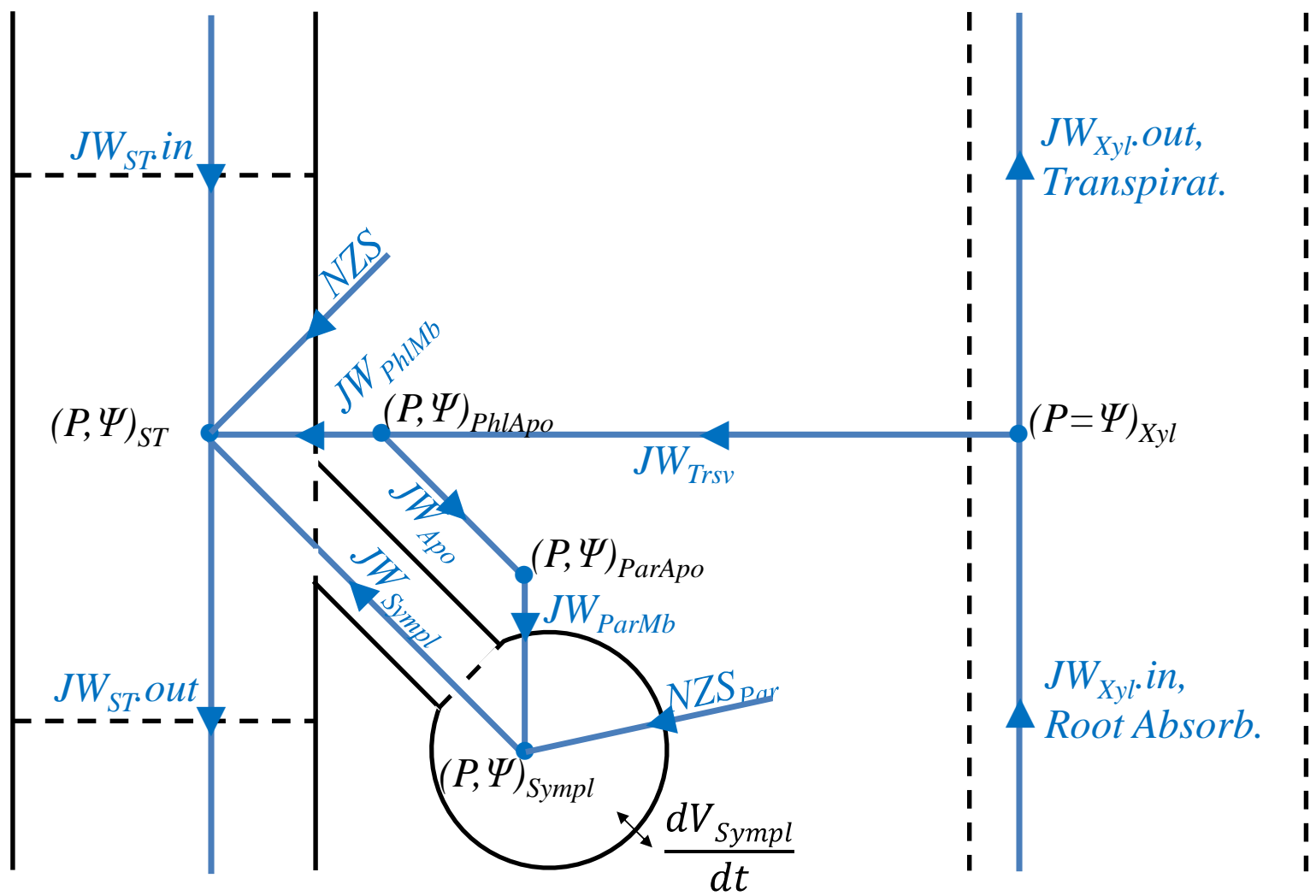

Phloem

sieve tube

\section{Lateral tissues}

Xylem

vessel

$$
\begin{aligned}
& \Delta P_{S T}=r_{S T} \cdot J W_{S T} \\
& \Psi_{S T}=P_{S T}+\Pi_{S T} \\
& \Psi_{\text {PhlApo }}=P_{\text {PhlApo }}+\Pi_{\text {PhlApo }} \\
& \Delta J W_{S T}=-N Z S-J W_{P h l M b}-J W_{S y m p l} \\
& \begin{aligned}
J W_{\text {Trsv }} & =J W_{\text {PhlMb }}+J W_{\text {Apo }} \\
P_{\text {Xyl }}-P_{\text {PhlApo }} & =r_{\text {Trsv }} \cdot J W_{\text {Trsv }}
\end{aligned} \\
& J W_{\text {Apo }}=J W_{\text {ParMb }} \\
& r_{X y l} \cdot J W_{X y l}=\Delta P_{X y l} \\
& \Psi_{\text {ParApo }}=P_{\text {ParApo }}+\Pi_{\text {ParApo }} \\
& \Psi_{\text {Sympl }}=P_{\text {Sympl }}+\Pi_{\text {Sympl }} \\
& P_{\text {Sympl }}-P_{S T}=r_{\text {Sympl }} \cdot J W_{\text {Sympl }} \\
& \Psi_{\text {PhlApo }}-\Psi_{S T}=r_{P h l M b} \cdot J W_{P h l M b} \\
& \text { Biological membrane } \\
& P_{\text {PhlApo }}-P_{\text {ParApo }}=r_{\text {Apo }} \cdot J W_{\text {Apo }} \\
& \Psi_{\text {ParApo }}-\Psi_{\text {Sympl }}=r_{\text {ParMb }} \cdot J W_{\text {ParMb }} \\
& d V_{\text {Sympl }} / d t=J W_{\text {ParMb }}-J W_{\text {Sympl }}+N Z S_{\text {Par }}
\end{aligned}
$$


$1 \quad$ Figure 4

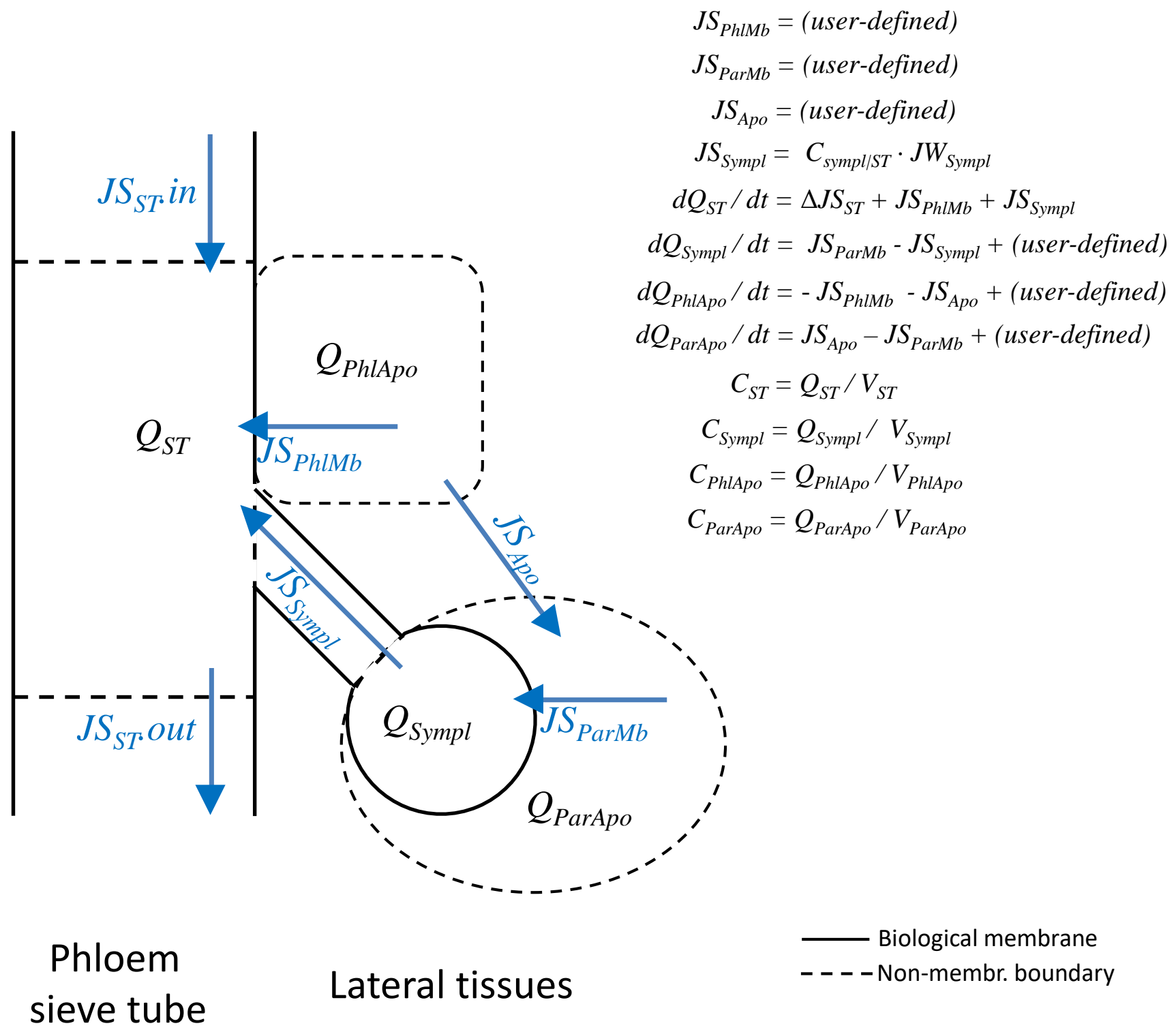

